\title{
On the Computational Power of Sigmoid versus Boolean Threshold Circuits
}

\author{
Extended Abstract
}

\author{
Wolfgang Maass * $\quad$ Georg Schnitger ${ }^{\dagger} \quad$ Eduardo D. Sontag ${ }^{\ddagger}$
}

\begin{abstract}
We examine the power of constant depth circuits with sigmoid (i.e. smooth) threshold gates for computing boolean functions. It is shown that, for depth 2 , constant size circuits of this type are strictly more powerful than constant size boolean threshold circuits (i.e. circuits with boolean threshold gates). On the other hand it turns out that, for any constant depth $d$, polynomial size sigmoid threshold circuits with polynomially bounded weights compute exactly the same boolean functions as the corresponding circuits with boolean threshold gates.
\end{abstract}

\section{Introduction}

Research on neural networks has led to the investigation of massively parallel computational models that consist of analog computational elements. Usually these analog computational elements are assumed to be smooth threshold gates, i.e. $\gamma$-gates for some nondecreasing differentiable function $\gamma: \mathbf{R} \rightarrow \mathbf{R}$. A $\gamma$-gate with weights $w_{1}, \ldots, w_{m} \in \mathbf{R}$ and threshold $t \in \mathbf{R}$ is defined to be a gate that computes the function $\left(x_{1}, \ldots, x_{m}\right) \mapsto \gamma\left(\sum_{i=1}^{m} w_{i} x_{i}-t\right)$ from $\mathbf{R}^{m}$ into R. A $\gamma$-circuit is defined as a directed acyclic circuit that consists of $\gamma$-gates. The most frequently considered special case of a smooth threshold circuit is the sigmoid threshold circuit, which is a $\sigma$-circuit for $\sigma: \mathbf{R} \rightarrow \mathbf{R}$ defined by $\sigma(x)=\frac{1}{1+\exp (-x)}$.

Smooth threshold circuits ( $\gamma$-circuits for "smooth" functions $\gamma$ ) have become the standard model for the investigation of learning on multi-layer artificial neural nets ([K],[HKP],[RM],[SS1],[SS2],[WK]). In fact, the most common learning algorithm for multi-layer neural nets, the Backwards-Propagation algorithm, can

*Institutes for Information Processing Graz, Graz University of Technology, Klosterwiesgasse 32, A-8010 Graz, Austria. E mail: maass@iicm.tu-graz.ac.at. Phone: (0316) 810063. Also affiliated with the University of nlinois at Chicago. Partially supported by NSF-CCR-8903398.

tDepartment of Computer Science, Penn State University. E-mail: georg@cs.psu.edu. Phone: (814) 865-1582. Partially supported by NSF-CCR-8805978 and AFOSR-87-0400.

'SYCON - Rutgers Center for Systems and Control, Department of Mathematics, Rutgers University. E-mail: sontag@hilbert.rutgers.edu. Phone: (908) 932-3072. Partially supported by Siemens Corporate Research and AFOSR-88-0235. only be implemented on $\gamma$-circuits for differentiable functions $\gamma$.

Another motivation for the investigation of smooth threshold circuits is the desire to explore simple models for the (very complicated) information processing mechanisms in neural systems of living organisms. In a first approximation one may view the current firing rate of a neuron as its current output ([S], [RM], [K]) The firing rates of neurons are known to change between a few and several hundred firings per second. Hence a smooth threshold gate provides a somewhat better computational model for a neuron than a digital element that has just two different output signals.

In this paper we examine the power of smooth threshold circuits for computing Boolean functions. In particular, we compare their power with that of boolean threshold circuits (i.e. $s$-circuits for the step function $s$, with $s(x)=1$ if $x \geq 0$ and $s(x)=0$ if $x<0)$. The most surprising result is the existence of a boolean function $F_{n}$, that can be computed by a large class of $\gamma$-circuits (containing $\sigma$-circuits) with small weights in depth 2 and size 5 (Theorem 2.2.), but which cannot be computed with any weight size by constant size boolean threshold circuits of depth 2 (Theorem 3.1). A witness for this difference in computational power is the boolean function $F_{n}$ with

$$
F_{n}(\vec{x}, \vec{y}):=\text { Majority }(\vec{x}) \oplus \text { Majority }(\vec{y}),
$$

where $\vec{x}$ and $\vec{y}$ are $n$-bit vectors.

The proof of this lower bound result for boolean threshold circuits (Theorem 3.1) is of independent interest. First, this proof demonstrates that the restriction method is not only useful in order to prove lower bounds for $A C^{0}$-circuits, but also for threshold circuits. Secondly, this proof exploits some previously unused potential in a standard tool for the analysis of threshold circuits: the $\varepsilon$-Discriminator Lemma of $[H M P S T]$. It is essential for our proof that the $\varepsilon$ Discriminator Lemma holds not just for the uniform distribution over the input space (as it is stated in [HMPST]), but for any distribution. Hence we have the freedom to construct such a distribution in a malicious manner, where we exploit specific "weak points" of the considered threshold circuit. This extra power of the (generalized) $\varepsilon$-Discriminator Lemma is crucial: in Remark 3.10 we show that its conventional version is insufficient for the proof of Theorem 3.1. 
In order to compute a boolean function on an analog computational device one has to adopt a suitable output convention (similar to the conventions that are used to carry out digital computations on realworld computers, which consists of non-digital computational elements such as transistors).

Definition $1.1 A \gamma$-circuit $C$ computes a boolean function $F:\{0,1\}^{n} \rightarrow\{0,1\}$ with separation $\varepsilon$ if there is some $t_{C} \in \mathbf{R}$ such that for any input $\left(x_{1}, \ldots, x_{n}\right) \in$ $\{0,1\}^{n}$ the output gate of $C$ outputs a value which is at least $t_{C}+\varepsilon$, if $F\left(x_{1}, \ldots, x_{n}\right)=1$ and at most $t_{C}-\varepsilon$ otherwise.

A computation without separation at the output gate appears to be less interesting, since then an infinitesimal change in the output of any $\gamma$-gate in the circuit may invert the output bit. Hence we consider in this paper computations on $\gamma$-circuits $C_{n}$ with separation at least $\frac{1}{p(n)}$ for some polynomial $p$ (where $n$ is the number of input bits of $C_{n}$ ). One nice feature of this convention is that, for Lipschitz bounded gate functions $\gamma$ and polynomial size $\gamma$-circuits $C_{n}$ of constant depth and with polynomially bounded weights, it allows a tolerance of $\frac{1}{\text { poly(n) }}$ for all $\gamma$-gates in $C_{n}$.

We will give in Theorem 4.1 a "separation boosting" result, which says that for any constant depth $d$ one may demand for polynomial size $\gamma$-circuits with polynomially bounded weights just as well a separation of size $\Omega(1)$ without changing the class of boolean functions that can be computed.

\section{Sigmoid Threshold Circuits for the XOR of Majorities}

We write (NL) for the following property of a function $\gamma: \mathbf{R} \rightarrow \mathbf{R}$,

(NL) There is some rational number $s$ so that:

1. $\gamma$ is differentiable on some open interval containing $s$, and

2. $\gamma^{\prime \prime}(s)$ exists and is nonzero.

Obviously the function $\sigma$ satisfies (NL)

Observe that property (NL) is basically the requirement that the function be nonlinear; for instance, if $\gamma^{\prime \prime}$ happens to be everywhere defined, then (NL) is precisely equivalent to $\gamma$ not being a linear function. The nonlinearity of $\gamma$ is obviously a necessary assumption for Theorem 2.2 , since otherwise a $\gamma$-circuit can only compute linear functions.

Without loss of generality, we will assume that

$$
c:=\frac{\gamma^{\prime \prime}(s)}{4}>0
$$

for some point $s$ as in the definition. If this value where to be negative, we simply replace $\gamma$ by $-\gamma$ in what follows.
Lemma 2.1 Assume that $\gamma$ satisfies (NL). Define the function

$$
\theta(x):=\gamma(x+s)+\gamma(-x+s) .
$$

Then, the function $\theta$ is even, and there exists some $\varepsilon>0$ so that the following property holds:

$$
\theta(a+h)-\theta(a) \geq c h^{2} \text { for all } a, h \in[0, \varepsilon] .
$$

Proof. Note that $\theta(-x)=\theta(x)$ directly from the definition, so $\theta$ is even. Moreover, $\theta$ is differentiable on some open interval containing $x=0$, because $\gamma$ is differentiable in a neighborhood of $s$, and evenness implies that $\theta^{\prime}(0)=0$. Observe also that $\theta^{\prime \prime}(0)$ exists, and in fact

$$
\theta^{\prime \prime}(0)=2 \gamma^{\prime \prime}(s)=8 c>0 .
$$

By definition of $\theta^{\prime \prime}(0)$ (just write $\theta^{\prime}(l)=\theta^{\prime}(0)+$ $\theta^{\prime \prime}(0) l+r(l)$ with $\lim _{l \rightarrow 0} \frac{r(l)}{l}=0$ ), there is some $\varepsilon>0$ so that

$$
\theta^{\prime}(l) \geq \frac{\theta^{\prime \prime}(0) l}{2}=4 c l
$$

for all each $l \in[0,2 \varepsilon]$. Because $\theta^{\prime}(l)>4 c l>0$ for $l>0$, it follows that $\theta$ is strictly increasing on $[0,2 \varepsilon]$. We are only left to prove that this $\varepsilon$ is so that the last property holds.

Pick any $a, h \in[0, \varepsilon]$. Assume that $h \neq 0$, as otherwise there is nothing to prove. As $a$ and $a+h$ are both in the interval $[0,2 \varepsilon]$, and $\theta$ is strictly increasing there, it follows that

$$
\theta(a+h)-\theta(a)>\theta(a+h)-\theta\left(a+\frac{h}{2}\right)
$$

and by the Mean Value Theorem this last expression equals $\theta^{\prime}(l) \frac{h}{2}$ for some $l \in\left(a+\frac{h}{2}, a+h\right)$.

Since $l<a+h \leq 2 \varepsilon$, we may apply inequality (1) to obtain

$$
\theta(a+h)-\theta(a)>2 c l h .
$$

The result now follows from the fact that $l>a+\frac{h}{2} \geq$ $\frac{h}{2}$.

Theorem 2.2 Assume that $\gamma: \mathbf{R} \rightarrow \mathbf{R}$ satisfies (NL). Then there exists for every $n \in \mathbf{N}$ a $\gamma$-circuit $C_{n}$ of depth 2 with 5 gates (and rational weights and thresholds of size $\mathrm{O}(1))$ that computes $F_{n}$ with separation $\Omega\left(1 / n^{2}\right)$.

Proof. With $\theta$ and $\varepsilon$ as in Lemma 2.1 one has $\theta(a)>\theta(b) \Leftrightarrow|a|>|b|$ for any $a, b \in[-\varepsilon+\varepsilon]$. Hence any two nonzero reals $u, v \in[-\varepsilon / 2,+\varepsilon / 2]$ have different sign if and only if $\theta(u-v)-\theta(u+v)>0$. Let $x_{1}, \ldots, x_{n}, y_{1}, \ldots, y_{n} \in\{0,1\}$ be arbitrary and set

$$
\begin{aligned}
& u:=\frac{\varepsilon}{2}\left(\frac{4\left(x_{1}+\ldots+x_{n}\right)-2 n+1}{4 n}\right), \\
& v:=\frac{\varepsilon}{2}\left(\frac{4\left(y_{1}+\ldots+y_{n}\right)-2 n+1}{4 n}\right) .
\end{aligned}
$$


Then we obtain

$$
\theta(u-v)-\theta(u+v)>0 \Leftrightarrow F_{n}(\vec{x}, \vec{y})=1 .
$$

Furthermore Lemma 2.1 implies that

$$
|\theta(u-v)-\theta(u+v)| \geq 4 c \cdot \min \left\{u^{2}, v^{2}\right\}=\Omega\left(1 / n^{2}\right) .
$$

Hence, we can achieve separation $\Omega\left(1 / n^{2}\right)$ by using a $\gamma$-gate on level two of circuit $C_{n}$ that checks whether $\theta(u-v)-\theta(u+v)>0$. Such a $\gamma$-gate exists: Since $\gamma^{\prime \prime}(s) \neq 0$, there is some $t$ with $\gamma^{\prime}(t) \neq 0$. Now transform $\theta(u-v)-\theta(u+v)$ into a suitable neighborhood of $t$ and choose a suitable rational approximation of $\theta(t)$ as threshold.

Corollary 2.3 Assume that $\gamma: \mathbf{R} \rightarrow \mathbf{R}$ satisfies (NL) and $\gamma$ is monotone. Then there exists for every $n \in \mathbb{N}$ a $\gamma$-circuit $C_{n}$ of depth 2 and size 5 (with rational weights and thresholds of size polynomial in $n$ ) that computes $F_{n}$ with separation $\Omega(1)$.

Proof. Multiply the weights of the $\gamma$-gate on level two of the circuit $C_{n}$ with $n^{2}$ and transform the threshold accordingly. In this way we can ensure that the weighted sum computed at the top gate has distance $\Omega(1)$ from its threshold.

Remark 2.4. For computations with real (rather than Boolean) inputs, there has been some work dealing with the differences in capabilities between sigmoidal and threshold devices; in particular [So] studies questions of interpolation and classification related to learnability (VC dimension).

\section{Boolean threshold gates are less powerful}

Theorem 3.1 No family $\left(C_{n} \mid n \in \mathbf{N}\right)$ of constant size boolean threshold circuits of depth 2 (with unrestricted weights and thresholds) can compute the function $F_{n}$.

Proof. Assume, by way of contradiction, that there exist such circuits $C_{n}$, each with at most $k^{\prime}$ gates on level one. We can demand that all weights are integers and that the level 2 gate has weights of absolute value at most $2^{O\left(k^{\prime} \log k^{\prime}\right)}([\mathrm{M}],[\mathrm{MT}])$. Thus we can assume, after appropriate duplication of level one gates, that the gate on level 2 has only weights from $\{-1,1\}$. Let $k$ be an upper bound on the resulting number of gates.

In the next section we use the restriction method to eliminate those gates on level one of $C_{n}$ whose weights for the $x_{i}\left(y_{i}\right)$ have drastically different sizes. It turns out that we cannot achieve this goal for all gates. For example, if all the weights $w_{i}$ (for the $x_{i}$ ) are much larger than the weights $u_{i}$ (for the $y_{i}$ ), then we can only limit the variance of the weights $w_{i}$ (see condition b. in Definition 3.2). Nevertheless, the restriction method allows us to "regularize" all bottom gates of
$C_{n}$ (see Lemma 3.4). In section 3.2 we show that the resulting regularized gates behave predictably for certain distributions (see Lemma 3.7). The argument concludes in section 3.3 with a non-standard application of the $\varepsilon$-Discriminator Lemma.

\subsection{The Restriction Method}

Our goal will be to fix certain inputs such that all bottom gates of $C_{n}$ will have a normal form as described in the following definition.

Definition 3.2. Let $G$ be a boolean threshold gate (with inputs $x_{1}, \ldots, x_{m}, y_{1}, \ldots, y_{m}$ ) that outputs 1 if and only if $\sum_{i=1}^{m} w_{i} x_{i}+\sum_{i=1}^{m} u_{i} y_{i} \geq t$. Assume that the numbering is such that

$$
\left|w_{1}\right| \leq \ldots \leq\left|w_{m}\right| \text { and }\left|u_{1}\right| \leq \ldots \leq\left|u_{m}\right| .
$$

We say that $G$ is $l$-regular if and only if all $w_{i}$ have the same sign (negative, zero, or positive) and all $u_{i}$ have the same sign. Additionally, one of the following conditions has to hold,

a. $G$ is constant.

b. $\forall i\left(\left|w_{i}\right| \geq m^{1 / 8}\left|u_{i}\right|\right)$ and $\left|w_{m}\right| \leq 60\left|w_{1}\right|$.

c. $\forall i\left(\left|u_{i}\right| \geq m^{1 / 8}\left|w_{i}\right|\right)$ and $\left|u_{m}\right| \leq 60\left|u_{1}\right|$.

d. $\left|w_{m}\right| \leq 30(1+l)\left|w_{1}\right|$ and $\left|u_{m}\right| \leq 30(1+l)\left|u_{1}\right|$.

First we will transform a single threshold gate to a regular gate.

Lemma 3.3 Let $G$ be an arbitrary threshold gate that outputs 1 if and only if

$$
\sum_{i=1}^{n} w_{i} x_{i}+\sum_{i=1}^{n} u_{i} y_{i} \geq t
$$

Then there are sets $M_{x} \subseteq\{1, \ldots, n\}$ and $M_{y} \subseteq$ $\{1, \ldots, n\}$ of size $\frac{n}{60}$ each and an assignment $\mathcal{A}:\left\{x_{i}\right.$ : $\left.i \notin M_{x}\right\} \cup\left\{y_{i}: i \notin M_{y}\right\} \rightarrow\{0,1\}$ such that

a. when values are assigned according to $\mathcal{A}, F_{n / 60}$ will be obtained as the corresponding subfunction of $F_{n}$, and

b. $G$, when restricted to the remaining free variables, is $n^{1 / 8}$-regular.

Proof. First we determine a set $M_{x}^{\prime} \subseteq\{1, \ldots, n\}$ of size $n / 3$ such that all $w_{i}$ (with $i \stackrel{x}{\in} M_{x}^{\prime}$ ) are either all positive, all negative or all zero. A set $M_{y}^{\prime} \subseteq\{1, \ldots, n\}$ of size $n / 3$ is chosen analogously to enforce the same property for the coefficients $u_{i}$ (with $i \in M_{y}^{\prime}$ ).

Set $m=n / 3$. After possibly renumbering the indices, we can assume that $M_{x}^{\prime}=M_{y}^{\prime}=\{1, \ldots, m\}$. We can also assume that $\left|w_{1}\right| \leq \ldots \leq\left|w_{m}\right|$ as well as $\left|u_{1}\right| \leq \ldots \leq\left|u_{m}\right|$. We define 


$$
\begin{aligned}
\mathcal{R} & :=\left\{1, \ldots, \frac{m}{4}\right\}, \mathcal{S}:=\left\{\frac{m}{4}+1, \ldots, \frac{3 m}{4}\right\} \\
\mathcal{T} & :=\left\{\frac{3 m}{4}+1, \ldots, m\right\}
\end{aligned}
$$

By assigning 1's to the $x_{i}$ 's with $i \in \mathcal{R}$ and 0 's to the $x_{i}$ 's with $i \in \mathcal{T}$ or vice versa, and by assigning 1 's to the $y_{i}$ 's with $i \in \mathcal{R}$ and 0 's to the $y_{i}$ 's with $i \in \mathcal{T}$ or vice versa, we obtain four partial assignments.

Let us now interpret $G$ as a threshold gate of the remaining variables $x_{i}(i \in \mathcal{S})$ and $y_{i}(i \in \mathcal{S})$. By choosing one of the four assignments, we can "move" the threshold of the resulting gate over a distance $d$ with

$$
d=\sum_{i \in \mathcal{T}}\left|w_{i}\right|-\sum_{i \in \mathcal{R}}\left|w_{i}\right|+\sum_{i \in \mathcal{T}}\left|u_{i}\right|-\sum_{i \in \mathcal{R}}\left|u_{i}\right|
$$

If for none of these four partial assignments the threshold gate $G$ gives constant output, we have

$$
d \leq \sum_{i \in \mathcal{S}}\left|w_{i}\right|+\sum_{i \in \mathcal{S}}\left|u_{i}\right|
$$

This implies that

$$
\begin{gathered}
\sum_{i \in \mathcal{T}}\left(\left|w_{i}\right|+\left|u_{i}\right|\right) \leq \sum_{i \in \mathcal{R} \cup \mathcal{S}}\left(\left|w_{i}\right|+\left|u_{i}\right|\right) . \\
\text { Set } \quad a=\sum_{i \in \mathcal{R} \cup \mathcal{S}}\left(\left|w_{i}\right|+\left|u_{i}\right|\right) /(3 m / 4) \text { and } \\
b=\sum_{i \in \mathcal{T}}\left(\left|w_{i}\right|+\left|u_{i}\right|\right) /(m / 4) .
\end{gathered}
$$

Then (2) implies for these "averages" of $\left|w_{i}\right|+\left|u_{i}\right|$ over $\mathcal{R} \cup \mathcal{S}$ respectively $\mathcal{T}$ that $b \leq 3 a$.

We subdivide the set $\mathrm{S}$ by introducing the sets

$$
\begin{aligned}
& \mathcal{P}=\left\{\frac{3 m}{4}-\frac{2 m}{10}+1, \ldots, \frac{3 m}{4}-\frac{m}{10}\right\} \\
& \mathcal{Q}=\left\{\frac{3 m}{4}-\frac{m}{10}+1, \ldots, \frac{3 m}{4}\right\} .
\end{aligned}
$$

Since $\left|w_{i}\right|+\left|u_{i}\right|$ is a non-decreasing function of $i$ we have for all $i \in \mathcal{R} \cup \mathcal{S}$ (and in particular for all $i \in \mathcal{P} \cup \mathcal{Q}$ )

$$
\left|w_{i}\right|+\left|u_{i}\right| \leq b \leq 3 a .
$$

Furthermore, we have for all $i \in \mathcal{P}$

$$
\left|w_{i}\right|+\left|u_{i}\right| \geq a / 10
$$

since otherwise $\left|w_{i}\right|+\left|u_{i}\right|<a / 10$ for all $i \in(\mathcal{R} \cup \mathcal{S})-$ $(\mathcal{P} \cup \mathcal{Q})$, and we would get

$$
\begin{aligned}
& \sum_{i \in \mathcal{R} \cup \mathcal{S}}\left(\left|w_{i}\right|+\left|u_{i}\right|\right)= \\
& \sum_{i \in(\mathcal{R} \cup \mathcal{S})-(\mathcal{P} \cup \mathcal{Q})}\left(\left|w_{i}\right|+\left|u_{i}\right|\right)+\sum_{i \in \mathcal{P} \cup \mathcal{Q}}\left(\left|w_{i}\right|+\left|u_{i}\right|\right) \leq
\end{aligned}
$$

$$
\begin{aligned}
& \left(\frac{3}{4}-\frac{2}{10}\right) m \cdot \frac{a}{10}+3 a \cdot \frac{2 m}{10}= \\
& m \cdot a\left(\frac{3}{4} \cdot \frac{1}{10}+\frac{2}{10}\left(3-\frac{1}{10}\right)\right)<\frac{3 m \cdot a}{4},
\end{aligned}
$$

which is a contradiction to the definition of $a$.

(3) and (4) jointly imply that

$$
\max _{i \in \mathcal{P} \cup \mathcal{Q}}\left(\left|w_{i}\right|+\left|u_{i}\right|\right) \leq 30 \min _{i \in \mathcal{P} \cup \mathcal{Q}}\left(\left|w_{i}\right|+\left|u_{i}\right|\right) .
$$

Case 1: $\forall i \in \mathcal{P}\left(\left|w_{i}\right| \geq m^{1 / 8}\left|u_{i}\right| \vee\left|u_{i}\right| \geq m^{1 / 8}\left|w_{i}\right|\right)$. We can find a subset $\mathcal{P}^{\prime} \subseteq \mathcal{P}$ of size $m / 20$ such that

$\forall i \in \mathcal{P}^{\prime}\left(\left|w_{i}\right| \geq m^{1 / 8}\left|u_{i}\right|\right)$ or $\forall i \in \mathcal{P}^{\prime}\left(\left|u_{i}\right| \geq m^{1 / 8}\left|w_{i}\right|\right)$.

In the former case, (5) implies that

$$
\begin{aligned}
\max _{i \in \mathcal{P}^{\prime}}\left|w_{i}\right| & \leq 30 \min _{i \in \mathcal{P}^{\prime}}\left(\left|w_{i}\right|+\left|u_{i}\right|\right) \\
& \leq 30\left(1+m^{-1 / 8}\right) \min _{i \in \mathcal{P}^{\prime}}\left|w_{i}\right| \\
& \leq 60 \min _{i \in \mathcal{P}^{\prime}}\left|w_{i}\right| .
\end{aligned}
$$

Set $M_{x}=M_{y}=\mathcal{P}^{\prime}$ and fix the remaining variables such that exactly half of the $x_{i}$ 's and half of the $y_{i}$ 's are 0 .

Analogously, in the latter case we obtain $\max _{i \in \mathcal{P}^{\prime}}\left|u_{i}\right| \leq$ $60 \min _{i \in \mathcal{P}^{\prime}}\left|u_{i}\right| . M_{x}$ and $M_{y}$ are obtained as above.

Case 2: Otherwise.

Then $\exists i_{0} \in \mathcal{P}\left(\left|w_{i_{0}}\right|<m^{1 / 8}\left|u_{i_{0}}\right| \wedge\left|u_{i_{0}}\right|<m^{1 / 8}\left|w_{i_{0}}\right|\right)$. We have for all $i \in \mathcal{Q}$ :

$$
\begin{aligned}
& \left|w_{i}\right|+\left|u_{i}\right| \leq 30\left(\left|w_{i_{0}}\right|+\left|u_{i_{0}}\right|\right) \\
& \leq \min \left\{30\left|w_{i_{0}}\right|\left(1+m^{1 / 8}\right), 30\left|u_{i_{0}}\right|\left(1+m^{1 / 8}\right)\right\} .
\end{aligned}
$$

Thus we have $\max _{i \in \mathcal{Q}}\left|w_{i}\right| \leq 30\left(1+m^{1 / 8}\right) \min _{i \in \mathcal{Q}}\left|w_{i}\right|$ and $\max _{i \in \mathcal{Q}}\left|u_{i}\right| \leq 30\left(1+m^{1 / 8}\right) \min _{i \in \mathcal{Q}}\left|u_{i}\right|$.

Choose $M_{x}$ to be an arbitrary subsets of $\mathcal{Q}$ of size $\frac{n}{60}$, set $M_{y}=M_{x}$ and fix the remaining variables in the same fashion as before.

If we perform the "regularization process" for all bottom gates of $C_{n}$, then we obtain

Lemma 3.4 There are sets $M_{x}, M_{y} \subseteq\{1, \ldots, n\}$ of size $m=\frac{n}{60^{k}}$ and there is an assignment $\mathcal{A}:\left\{x_{i}: i \notin\right.$ $\left.M_{x}\right\} \cup\left\{y_{i}: i \notin M_{y}\right\} \rightarrow\{0,1\}$ such that

a. when values are assigned according to $\mathcal{A}, F_{m}$ will be obtained as the corresponding subfunction of $F_{n}$, and

b. all level one gates of $C_{n}$, when restricted to the free variables, are $n^{1 / 8}$-regular. 
Proof. Apply Lemma 3.3 successively to each of the $k$ level one gates of $C_{n}$. Let $M_{x}$ be the set of indices of those variables $x_{i}$ which did not receive a value during the processing of all gates by Lemma 3.3. $M_{y}$ is defined analogously.

$\mathcal{A}$ is the union of all partial assignments that have been made in this process.

We write $D_{n}$ for the circuit that results from $C_{n}$ by the restriction of Lemma 3.4. Observe that $D_{n}$ computes the function $F_{m}$ (for $m=\frac{n}{60^{k}}$ ).

\subsection{The Likely Behavior of a Threshold Gate}

In this section we will exploit the result of our regularization process. In particular, in Lemma 3.7 , we will show that, for the input distribution defined below, a weighted sum with small variance in weight sizes "almost" behaves as if all the weights were identical.

For the integer $s, 1 \leq s \leq m$, set $U(s)=\{\vec{x} \epsilon$ $\left.\{0,1\}^{m}: \sum_{i=1}^{m} x_{i}=s\right\} . X(s)$ is the random variable which assigns to each $\vec{x} \in U(s)$ the value $\sum_{i=1}^{m} w_{i} x_{i}$; all elements of $U(s)$ are equally likely. Obviously $E(X(s))=\frac{s}{m} \sum_{i=1}^{m} w_{i}$

In the following, we will assume that the $w_{i}$ 's are either all positive or all negative.

Proposition 3.5 Set $W=\sum_{i=1}^{m} w_{i}^{2}$ and $g=\max \left\{\frac{\left|w_{i}\right|}{\left|w_{j}\right|}: 1 \leq i, j \leq m\right\}$. Then

$$
W \leq \frac{m}{s^{2}} g^{2} \cdot E(X(s))^{2} .
$$

Proof. Set $M I N=\min \left\{\left|w_{i}\right|: 1 \leq i \leq m\right\}$. We get

$$
W \leq \frac{1}{m}\left(m^{2} \cdot g^{2} \cdot M I N^{2}\right) \text {. }
$$

Also, $E(X(s))^{2}=\left(\frac{s}{m} \cdot \sum_{i=1}^{m} w_{i}\right)^{2} \geq\left(\frac{s}{m} m \cdot M I N\right)^{2}$. Thus

$$
m^{2} \cdot M I N^{2} \leq\left(\frac{m}{s}\right)^{2} \cdot E(X(s))^{2} .
$$

If we replace $m^{2} \cdot M I N^{2}$ in (6) according to (7), we get

$$
W \leq \frac{m}{s^{2}} \cdot g^{2} \cdot E(X(s))^{2}
$$

Proposition $3.6 \operatorname{Var}(X(s)) \leq \frac{s}{m} W$

Proof. We have $\operatorname{Var}(X(s))=E\left(X(s)^{2}\right)-E(X(s))^{2}$. Also, $E\left(X(s)^{2}\right)=$

$\frac{1}{\left(\begin{array}{c}m \\ s\end{array}\right)} \sum_{x \in U(s)}\left(\sum_{i=1}^{m} w_{i} x_{i}\right)^{2}=$

$\frac{1}{\left(\begin{array}{c}m \\ s\end{array}\right)}\left(\sum_{x \in U(s)} \sum_{i=1}^{m} w_{i}^{2} x_{i}+2 \sum_{\vec{x} \in U(s)} \sum_{1 \leq i<j \leq m} w_{i} w_{j} x_{i} x_{j}\right)=$ $\frac{1}{\left(\begin{array}{c}m \\ s\end{array}\right)}\left(\sum_{i=1}^{m} w_{i}^{2} \sum_{z \in U(s)} x_{i}+2 \sum_{1 \leq i<j \leq m} w_{i} w_{j} \sum_{z \in \in U(s)} x_{i} x_{j}\right)=$ $\frac{s}{m} \cdot \sum_{i=1}^{m} w_{i}^{2}+\frac{2 s(s-1)}{m(m-1)} \sum_{1 \leq i<j \leq m} w_{i} w_{j}$

Furthermore, $E(X(s))^{2}=\left(\frac{1}{\left(\begin{array}{c}m \\ s\end{array}\right)} \sum_{x \in U(s)} \sum_{i=1}^{m} w_{i} x_{i}\right)^{2}$

$=\left(\frac{1}{\left(\begin{array}{c}m \\ s\end{array}\right)} \sum_{i=1}^{m} w_{i} \sum_{x \in U(s)} x_{i}\right)^{2}=\left(\frac{s}{m} \sum_{i=1}^{m} w_{i}\right)^{2}$

$=\frac{s^{2}}{m^{2}} \sum_{i=1}^{m} w_{i}^{2}+\frac{2 s^{2}}{m^{2}} \sum_{1 \leq i<j \leq m} w_{i} w_{j}$.

Summarizing, we obtain

$$
\operatorname{Var}(X(s)) \leq\left(\frac{s}{m}-\frac{s^{2}}{m^{2}}\right) \sum_{i=1}^{m} w_{i}^{2} \leq \frac{s}{m} W .
$$

Lemma 3.7 If $\max \left\{\frac{\left|w_{i}\right|}{\left|w_{j}\right|}: 1 \leq i, j \leq m\right\}=O\left(m^{1 / 8}\right)$, then

$$
\operatorname{Pr}\left(|X(s)-E(X(s))| \geq \frac{|E(X(s))|}{m^{1 / 4}}\right)=O\left(\frac{m^{3 / 4}}{s}\right) .
$$

Proof. By Chebyshev's inequality, we get for any $t$ $(t>0)$

$$
\operatorname{Pr}(|X(s)-E(X(s))| \geq t) \leq \frac{\operatorname{Var}(X(s)}{t^{2}}
$$

Thus, for $\left.t=\frac{|E(X(s))|}{m^{1 / 4}}\right)$, we obtain

$$
\begin{gathered}
\operatorname{Pr}\left(|X(s)-E(X(s))| \geq \frac{|E(X(s))|}{m^{1 / 4}}\right) \\
\leq \frac{\operatorname{Var}(X(s)) \cdot m^{1 / 2}}{E(X(s))^{2}} .
\end{gathered}
$$

Proposition 3.6 implies

$$
\frac{\operatorname{Var}(X(s)) \cdot m^{1 / 2}}{E(X(s))^{2}} \leq \frac{s \cdot W \cdot m^{1 / 2}}{m \cdot E(X(s))^{2}},
$$

and with Proposition 3.5

$$
\frac{s \cdot W \cdot m^{1 / 2}}{m \cdot E(X(s))^{2}} \leq \frac{m}{s} g^{2} m^{-1 / 2}=O\left(\frac{m}{s} m^{-1 / 4}\right) .
$$




\subsection{A Non-standard Application of the Discriminator Lemma}

Let $G$ be some boolean threshold gate with weights $w_{1}, \ldots, w_{m}, u_{1}, \ldots, u_{m}$ and threshold $t$. Set

$$
a:=\frac{\sum_{i=1}^{m} w_{i}}{m} \text { and } b:=\frac{\sum_{i=1}^{m} u_{i}}{m}
$$

With $G$ we can thus associate the two-dimensional threshold function $a x+b y \geq t$. Similarly, with $F_{m}$ we associate the two-dimensional function $F$ : $\{0, \ldots, m\}^{2} \rightarrow\{0,1\}$, where $F(x, y)=1$ if and only if

$$
\left(x \geq \frac{m}{2} \wedge y<\frac{m}{2}\right) \vee\left(x<\frac{m}{2} \wedge y \geq \frac{m}{2}\right) .
$$

Let $L$ be the line $a x+b y=t$ in $\mathbf{R}^{2}$ (where $t$ is the threshold of $G)$. Let $x^{\prime}\left(y^{\prime}\right)$ be the x-coordinate ( $\mathrm{y}$ coordinate) of the intersection of $L$ and $y=\frac{m}{2}(x=$ $\left.\frac{m}{2}\right)$. Set $x^{\prime}=\infty\left(y^{\prime}=\infty\right)$ if the line $L$ is horizontal (vertical). We define

$$
D(G):=\min \left\{\left|x^{\prime}-\frac{m}{2}\right|,\left|y^{\prime}-\frac{m}{2}\right|\right\} .
$$

Proposition 3.8 Let $r$ be an integer with $0 \leq r \leq \frac{m}{2}$ and let $U_{r}$ be the uniform distribution over $V_{r}=\left\{\frac{m}{2}-r, \ldots, \frac{m}{2}+r\right\}$. Then

$$
\begin{aligned}
\operatorname{Pr}_{U_{r} \times U_{r}}[(x, y) & \left.\in V_{r}^{2} \mid a x+b y \geq t \wedge F(x, y)=1\right\} \\
& \leq \frac{1}{2}+\frac{D(G)+1}{2 r+1} .
\end{aligned}
$$

Proof. Let $X$ be the area enclosed by the two lines $a x+b y=t$ and $a x+b y=(a+b) \cdot \frac{m}{2}$. (The latter is the line through $\left(\frac{m}{2}, \frac{m}{2}\right)$.) Intersect $X$ with the set $V_{r}^{2}$ and call the intersection $X_{r}$.

Let us assume that $D(G)=\left|x^{\prime}-\frac{m}{2}\right|$. Then $X_{r}$ will contain at most $D(G)+1$ points per row of $V_{r}^{2}$. Thus $\left|X_{r}\right| \leq(2 r+1) \cdot(D(G)+1)$.

On the other hand, the halfspace $a x+b y \geq(a+b)$. $\frac{m}{2}$ contains exactly one half of all the elements of the set $\left\{(x, y) \in V_{r}^{2} \mid F(x, y)=1\right\}$.

Let us consider the case that all weights $w_{i}$ are identical and all weights $u_{i}$ are identical. If $D(G)$ is "small", then $G$ will not show any significant advantage in predicting $F$ for a subcollection of the $\frac{m}{2}+1$ distributions $U_{r}$ mentioned in Proposition 3.8. If on the other hand $D(G)$ is large (say proportional to $m$ ), then we can trivialize $G$ by choosing a distribution with a small value for $r$.

Our goal is to carry out a similar argument for arbitrary gates $G$. Consequently we introduce a collection $Q_{r}$ of distributions over $\{0,1\}^{m}$ with

$$
Q_{r}(\vec{x})=\left\{\begin{array}{cc}
0, & \text { if }\left|\sum_{i=1}^{m} x_{i}-\frac{m}{2}\right|>r \\
\frac{1}{(2 r+1) \cdot\left(\sum_{i=1}^{m} x_{i}\right)}, & \text { otherwise. }
\end{array}\right.
$$

Note that the probability of a string only depends on its number of ones. The appropriate value for the parameter $r$ will be determined later.

Finally we define for the considered threshold gate $G$ with input variables $x_{1}, \ldots, x_{m}, y_{1}, \ldots, y_{m}$, $A D V_{r}(G):=\operatorname{Pr}_{Q_{r} \times Q_{r}}\left[G(\vec{x}, \vec{y})=1 \mid F_{m}(\ddot{x}, \vec{y})=1\right]-$ $\operatorname{Pr}_{Q_{r} \times Q_{r}}\left[G(\vec{x}, \vec{y})=1 \mid F_{m}(\vec{x}, \vec{y})=0\right]$.

Lemma 3.9. Set $m=\frac{n}{60^{k}}$. Assume that the boolean threshold gate $G$ with input variables $x_{1}, \ldots, x_{m}, y_{1}, \ldots y_{m}$ is $n^{1 / 8}$-regular, and that $n$ is sufficiently large. Furthermore assume that the natural number $r \in\left[m^{31 / 32}, \frac{m}{4}\right]$ satisfies

$$
D(G) \leq r /(64 k) \text { or } D(G) \geq 4 r .
$$

Then

$$
\left|A D V_{r}(G)\right| \leq \frac{1}{2 k}
$$

Proof.

Case 1: $D(G) \leq \frac{r}{64 \cdot k}$.

We know that $G$ is $n^{1 / 8}$-regular. We proceed by examining the three different cases (see Definition 3.2.).

Case 1.1: $\forall i\left(\left|w_{i}\right| \geq m^{1 / 8}\left|u_{i}\right|\right)$ and $\left|w_{m}\right| \leq 60\left|w_{1}\right|$.

This implies that $|a| \geq m^{1 / 8} \cdot|b|$. Hence the line $L$ is very "steep". We have in this case,

$$
\max \{x \in[0, m]: \exists y \in[0, m]((x, y) \in L)\}-
$$

$\min \{x \in[0, m]: \exists y \in[0, m]((x, y) \in L)\} \leq m^{7 / 8}$.

Thus, the set $\left\{x \in[0, m]: \exists x^{\prime}, y^{\prime} \in[0, m]\left(\left|x-x^{\prime}\right| \leq\right.\right.$ $\left.m^{3 / 4} \wedge\left(x^{\prime}, y^{\prime}\right) \in L\right\}$ is contained in an interval of length $m^{7 / 8}+2 m^{3 / 4}+1 \leq 3 \cdot m^{7 / 8}$. This implies that

$$
\begin{aligned}
\mid\{(x, y) \in & \left.\left\{\frac{m}{2}-r, \ldots, \frac{m}{2}+r\right\}^{2}: P(x, y)\right\} \mid \\
& \leq 3 m^{7 / 8} \cdot m=3 m^{15 / 8}
\end{aligned}
$$

where $P(x, y)$ is equivalent to

$$
(a x+b y<t) \wedge
$$

$\exists\left(x^{\prime}, y^{\prime}\right) \in[0, m]^{2}\left(\left(a x^{\prime}+b y^{\prime} \geq t\right) \wedge\left(\left|x-x^{\prime}\right| \leq m^{3 / 4}\right)\right)$.

As a first step towards estimating $A D V_{r}(G)$ we consider the set

$$
S=\left\{(\vec{x}, \vec{y}) \in U: G(\vec{x}, \vec{y})=1 \wedge F_{m}(\vec{x}, \vec{y})=1\right\}
$$

where $U:=$

$$
\left\{(\vec{x}, \vec{y}) \in\{0,1\}^{2 m}: \frac{m}{2}-r \leq \sum_{i=1}^{m} x_{i}, \sum_{i=1}^{m} y_{i} \leq \frac{m}{2}+r\right\} \text {. }
$$

One shows that $S$ is contained in the following two sets, 


$$
\begin{gathered}
S_{1}=\{(\vec{x}, \vec{y}) \in U: \\
\left.\left|\sum_{i=1}^{m} w_{i} x_{i}-\left(\sum_{i=1}^{m} x_{i} \cdot \sum_{i=1}^{m} w_{i}\right) / m\right| \geq\left(\sum_{i=1}^{m} x_{i} \cdot \sum_{i=1}^{m} w_{i}\right) / m^{5 / 4}\right\}
\end{gathered}
$$

and $S_{2}=\left\{(\vec{x}, \vec{y}) \in U: F_{m}(\vec{x}, \vec{y})=1 \wedge Q(\vec{x}, \vec{y})\right\}$, where $Q(\vec{x}, \vec{y})$ is equivalent to

$$
\begin{aligned}
& \exists \overrightarrow{x^{\prime}}, \overrightarrow{y^{\prime}} \in\{0,1\}^{m}\left(\left|\sum_{i=1}^{m} x_{i}^{\prime}-\sum_{i=1}^{m} x_{i}\right| \leq m^{3 / 4} \wedge\right. \\
& \left.a \cdot \sum_{i=1}^{m} x_{i}^{\prime}+b \cdot \sum_{i=1}^{m} y_{i}^{\prime} \geq t\right) .
\end{aligned}
$$

Intuitively, the set $S_{1}$ consists of all those inputs (that are relevant for $Q_{r}$ ) on which our approximation of $G$ by $a x+b y \geq t$ fails. We will show later that this set has small probability. $S_{2}$ on the other hand is the collection of all relevant inputs on which the approximation (in a quite liberal sense) succeeds.

Let us verify the inclusion $S \subseteq S_{1} \cup S_{2}$. Fix any $(\vec{x}, \vec{y}) \in S-S_{1}$. We then have $\sum_{i=1}^{m} w_{i} x_{i}+u_{i} y_{i} \geq t$ and

$$
\begin{aligned}
\left|\sum_{i=1}^{m} w_{i} x_{i}-a \cdot \sum_{i=1}^{m} x_{i}\right| & \leq\left(\sum_{i=1}^{m} x_{i} \cdot|a|\right) / m^{1 / 4} \\
& \leq m^{3 / 4} \cdot|a| .
\end{aligned}
$$

We need to find vectors $\overrightarrow{x^{\prime}}, \overrightarrow{y^{\prime}}$ according to the definition of set $S_{2}$.

If $a \geq 0$, we pick some $\overrightarrow{x^{\prime}}$ such that $\sum_{\substack{i=1 \\ 3 m / 4}}^{m} x_{i}^{\prime}=\sum_{i=1}^{m} x_{i}+$ $m^{3 / 4}$. This is possible, since $U \subseteq \bigcup_{i=m / 4}^{3 m / 4}\{0,1\}^{i}$. We then have with (9) $a \cdot \sum_{i=1}^{m} x_{i}^{\prime} \geq \sum_{i=1}^{m} w_{i} x_{i}$.

If $a<0$, we pick some $\overrightarrow{x^{\prime}}$ such that $\sum_{i=1}^{m} x_{i}^{\prime}=\sum_{i=1}^{m} x_{i}-$ $m^{3 / 4}$. We then have $a \cdot \sum_{i=1}^{m} x_{i}^{\prime}=a \cdot \sum_{i=1}^{m} x_{i}-a \cdot m^{3 / 4}=$ $a \cdot \sum_{i=1}^{m} x_{i}+|a| \cdot m^{3 / 4} \geq \sum_{i=1}^{m} w_{i} x_{i}$.

Furthermore, we pick some vector $\overrightarrow{y^{\prime}}$ with $b \cdot \sum_{i=1}^{m} y_{i}^{\prime} \geq$ $\sum_{i=1}^{m} u_{i} y_{i}$ according to the following procedure: if all components of $u_{i}$ are positive or all components are zero, then set $\overrightarrow{y^{\prime}}=(1, \ldots, 1)$. Otherwise all components are negative and we set $\overrightarrow{y^{\prime}}=(0, \ldots, 0)$.
This concludes our proof of inclusion, since property $Q(\vec{x}, \vec{y})$ holds for the pair $\left(\overrightarrow{x^{\prime}}, \overrightarrow{y^{\prime}}\right)$.

It is obvious that $\operatorname{Pr}_{Q_{r} \times Q_{r}}\left[S_{1} \mid F_{m}(\vec{x}, \vec{y})=1\right] \leq$ $2 \cdot \operatorname{Pr}_{Q_{r} \times Q_{r}}\left[S_{1}\right]$. If we apply Lemma 3.7 for $s \bar{\epsilon}$ $\left[\frac{m}{2}-r, \frac{m}{2}+r\right] \subseteq\left[\frac{m}{4}, \frac{3 m}{4}\right]$, we obtain $\operatorname{Pr}_{Q_{r} \times Q_{r}}\left[S_{1}\right]=$ $O\left(m^{-1 / 4}\right)$.

In order to give an upper bound on $\operatorname{Pr}_{Q_{r} \times Q_{r}}\left[S_{2}\right]$ we observe that $S_{2} \subseteq S_{3} \cup S_{4}$, where

$$
\begin{gathered}
S_{3}=\left\{(\vec{x}, \vec{y}):\left(F_{m}(\vec{x}, \vec{y})=1\right) \wedge\right. \\
\left.\left(a \cdot \sum_{i=1}^{m} x_{i}+b \cdot \sum_{i=1}^{m} y_{i} \geq t\right)\right\} \\
S_{4}=\left\{(\vec{x}, \vec{y}):\left(F_{m}(\vec{x}, \vec{y})=1\right) \wedge\right. \\
\left.\left(a \cdot \sum_{i=1}^{m} x_{i}+b \cdot \sum_{i=1}^{m} y_{i}<t\right) \wedge R(\vec{x}, \vec{y})\right\} .
\end{gathered}
$$

$R(\vec{x}, \vec{y})$ is equivalent to

$$
\begin{gathered}
\exists \overrightarrow{x^{\prime}}, \overrightarrow{y^{\prime}}\left(\left|\sum_{i=1}^{m} x_{i}-\sum_{i=1}^{m} x_{i}^{\prime}\right| \leq m^{3 / 4} \wedge\right. \\
\left.a \cdot \sum_{i=1}^{m} x_{i}^{\prime}+b \cdot \sum_{i=1}^{m} y_{i}^{\prime} \geq t\right) .
\end{gathered}
$$

It follows from Proposition 3.8 that

$\operatorname{Pr}_{Q_{r} \times Q_{r}}\left[S_{3} \mid F_{m}(\vec{x}, \vec{y})=1\right] \leq \frac{1}{2}+\frac{D(G)+1}{2 r+1}$.

Also, it is obvious that

$\operatorname{Pr}_{Q_{r} \times Q_{r}}\left[S_{4} \mid F_{m}(\vec{x}, \vec{y})=1\right] \leq 2 \cdot \operatorname{Pr}_{Q_{r} \times Q_{r}}\left[S_{4}\right]$.

Furthermore, by (8), $\operatorname{Pr}_{Q_{r} \times Q_{r}}\left[S_{4}\right] \leq \frac{3 \cdot m^{15 / 8}}{(2 r+1)^{2}}$.

Thus we have

$\operatorname{Pr}_{Q_{r} \times Q_{r}}\left[S \mid F_{m}(\vec{x}, \vec{y})=1\right]$

$$
\begin{aligned}
& \leq \operatorname{Pr}_{Q_{r} \times Q_{r}}\left[S_{1} \cup S_{3} \cup S_{4} \mid F_{m}(\vec{x}, \vec{y})=1\right] \\
& \leq O\left(m^{-1 / 4}\right)+\frac{1}{2}+\frac{D(G)+1}{2 r+1}+\frac{6 m^{15 / 8}}{(2 r+1)^{2}} \\
& \leq \frac{1}{2}+\frac{1}{64 k}+O\left(m^{-1 / 16}\right) .
\end{aligned}
$$

We will obtain the same upper bound for the probability of

$$
S^{\prime}=\left\{(\vec{x}, \vec{y}) \in U: G(\vec{x}, \vec{y})=0 \wedge F_{m}(\vec{x}, \vec{y})=1\right\} .
$$

Thus, since

$$
\operatorname{Pr}_{Q_{r} \times Q_{r}}\left[S \mid F_{m}=1\right]+\operatorname{Pr}_{Q_{r} \times Q_{r}}\left[S^{\prime} \mid F_{m}=1\right]=1 \text {, }
$$

we get

$\left|\operatorname{Pr}_{Q_{r} \times Q_{r}}\left[S \mid F_{m}(\vec{x}, \vec{y})=1\right]-\frac{1}{2}\right| \leq \frac{1}{64 k}+O\left(m^{-1 / 16}\right)$.

One shows analogously for $T=\{(\vec{x}, \vec{y}) \in U$ : $\left.G(\vec{x}, \vec{y})=1 \wedge F_{m}(\vec{x}, \vec{y})=0\right\}$ that

$\left|\operatorname{Pr}_{Q_{r} \times Q_{r}}\left[T \mid F_{m}(\vec{x}, \vec{y})=0\right]-\frac{1}{2}\right| \leq \frac{1}{64 k}+O\left(m^{-1 / 16}\right)$. 
Thus, $\left|A D V_{r}(G)\right| \leq \frac{1}{32 k}+O\left(m^{-1 / 16}\right)$.

Case 1.2: $\forall i\left(\left|u_{i}\right| \geq m^{1 / 8}\left|w_{i}\right|\right)$ and $\left|u_{m}\right| \leq 60\left|u_{1}\right|$. The argument is analogous to Case 1.1 .

Case 1.3: $\left|w_{m}\right| \leq 30\left(1+n^{1 / 8}\right)\left|w_{1}\right|$ and $\left|u_{m}\right| \leq 30(1+$ $\left.n^{1 / 8}\right)\left|u_{1}\right|$.

We first observe that the set $S$ is contained in the union of the sets $S_{1}^{\prime}$ and $S_{2}^{\prime}$, where

$S_{1}^{\prime}=\left\{(\vec{x}, \vec{y}) \in U: P^{\prime}(\vec{x}, \vec{y})\right\}$, and $P^{\prime}(\vec{x}, \vec{y})$ is equivalent to

$$
\begin{aligned}
& \left(\left|\sum_{i=1}^{m} w_{i} x_{i}-a \cdot \sum_{i=1}^{m} x_{i}\right| \geq \frac{|a| \cdot \sum_{i=1}^{m} x_{i}}{m^{1 / 4}}\right) \vee \\
& \left(\left|\sum_{i=1}^{m} u_{i} y_{i}-b \cdot \sum_{i=1}^{m} y_{i}\right| \geq \frac{|b| \cdot \sum_{i=1}^{m} y_{i}}{m^{1 / 4}}\right)
\end{aligned}
$$

$S_{2}^{\prime}=\left\{(\vec{x}, \vec{y}) \in U: F_{m}(\vec{x}, \vec{y})=1 \wedge Q^{\prime}(\vec{x}, \vec{y})\right\}$, and $Q^{\prime}(\vec{x}, \vec{y})$ is equivalent to

$$
\begin{gathered}
\exists \overrightarrow{x^{\prime}}, \overrightarrow{y^{\prime}} \in\{0,1\}^{m}\left(\left|\sum_{i=1}^{m} x_{i}^{\prime}-\sum_{i=1}^{m} x_{i}\right| \leq m^{3 / 4} \wedge\right. \\
\left.\left|\sum_{i=1}^{m} y_{i}^{\prime}-\sum_{i=1}^{m} y_{i}\right| \leq m^{3 / 4} \wedge a \cdot \sum_{i=1}^{m} x_{i}^{\prime}+b \cdot \sum_{i=1}^{m} y_{i}^{\prime} \geq t\right) .
\end{gathered}
$$

Lemma 3.7 implies that $\operatorname{Pr}_{Q_{r} \times Q_{r}}\left[S_{1}^{\prime} \mid F_{m}(\vec{x}, \vec{y})=1\right] \leq$ $2 \cdot \operatorname{Pr}_{Q_{r} \times Q_{r}}\left[S_{1}^{\prime}\right]=O\left(m^{-1 / 4}\right)$.

With an argument analogous to Case 1.1 we get $S_{2}^{\prime} \subseteq$ $S_{3} \cup S_{4}^{\prime}$ where

$$
\begin{gathered}
S_{4}^{\prime}=\left\{(\vec{x}, \vec{y}):\left(F_{m}(\vec{x}, \vec{y})=1\right) \wedge\right. \\
\left.\left(a \cdot \sum_{i=1}^{m} x_{i}+b \cdot \sum_{i=1}^{m} y_{i}<t\right) \wedge R^{\prime}(\vec{x}, \vec{y})\right\} .
\end{gathered}
$$

$R^{\prime}(\vec{x}, \vec{y})$ is equivalent to

$$
\begin{gathered}
\exists \overrightarrow{x^{\prime}}, \overrightarrow{y^{\prime}}\left(\left|\sum_{i=1}^{m} x_{i}-\sum_{i=1}^{m} x_{i}^{\prime}\right| \leq m^{3 / 4} \wedge\right. \\
\left.\left|\sum_{i=1}^{m} y_{i}-\sum_{i=1}^{m} y_{i}^{\prime}\right| \leq m^{3 / 4} \wedge a \cdot \sum_{i=1}^{m} x_{i}^{\prime}+b \cdot \sum_{i=1}^{m} y_{i}^{\prime} \geq t\right) .
\end{gathered}
$$

We have already shown that

$$
\operatorname{Pr}_{Q_{r} \times Q_{r}}\left[S_{3} \mid F_{m}(\vec{x}, \vec{y})=1\right] \leq \frac{1}{2}+\frac{D(G)+1}{2 r+1} .
$$

Furthermore, it is obvious that

$$
\operatorname{Pr}_{Q_{r} \times Q_{r}}\left[S_{4}^{\prime} \mid F_{m}(\vec{x}, \vec{y})=1\right] \leq \frac{4 \cdot m \cdot m^{3 / 4}}{(2 r+1)^{2}} .
$$

The remaining argument is now analogous to Case 1.1.
Case 2: $D \geq 4 r$.

The analysis is now far simpler. The probability of the set $S_{1}$ (resp. $S_{1}^{\prime}$ ) is computed as before. As for $S_{3}$ we now get

$$
\operatorname{Pr}_{Q_{r} \times Q_{r}}\left[S_{3} \mid F_{m}(\vec{x}, \vec{y})=1\right] \in\{0,1\} .
$$

For $S_{4}$ we obtain

$$
\operatorname{Pr}_{Q_{r} \times Q_{r}}\left[S_{4} \mid F_{m}(\vec{x}, \vec{y})=1\right]=0 .
$$

The same applies to $S_{4}^{\prime}$. This follows, since the set $U$ will be entirely contained in one of the halfspaces of $\left\{(\vec{x}, \vec{y}): a \cdot \sum_{i=1}^{m} x_{i}+b \cdot \sum_{i=1}^{m} y_{i}=t\right\}$

In order to prove Theorem 3.1 we observe that for sufficiently large $n$ we can find $r$ such that for each of the at most $k$ gates $G$ on level one of $D_{n}$ :

$$
D(G) \leq \frac{r}{64 k} \text { or } D(G) \geq 4 r .
$$

(A value for $r$ can be found whenever $k$ is bounded from above by the number of possible " $r$-intervals". This is the case, provided $k \leq c \cdot \log _{k}(m)$ for a suitably small constant $c$. This in turn is satisfied for $k \leq$ $d \cdot \frac{\log n}{\log \log n}$ for a suitably small constant $d$.)

The $\varepsilon$-Discriminator Lemma of [HMPST] can be generalized to hold for any distribution over the input space. We apply it here to the distribution $Q_{r} \times Q_{r}$ over the input space $\{0,1\}^{2 m}$ of the circuit $D_{n}$ (which computes the function $F_{m}$ ).

Since the weights of the gate on level two of $D_{n}$ are from $\{-1,1\}$, we get $\left|A D V_{r}(G)\right| \geq \frac{1}{k}$ for some gate $G$ on level one of $D_{n}$. But this contradicts Lemma 3.5.

Thus we get a lower bound of $\Omega\left(\frac{\log n}{\log \log n}\right)$ for the size of depth 2 threshold circuits (with weights from $\{-1,1\}$ for the top gate) computing $F_{n}$. For unrestricted threshold circuits our lower bound will be $\Omega\left(\frac{\log \log n}{\log \log \log n}\right)([\mathrm{M}],[\mathrm{MT}])$.

Remark 3.10 It is not possible to prove Theorem 3.1 with the customary version of the $\varepsilon$-Discriminator Lemma, where one considers the uniform distribution over the input space. Consider for example the threshold gate $G$ defined by

$G\left(x_{1}, \ldots x_{n}, y_{1}, \ldots y_{n}\right)=1 \Leftrightarrow \sum_{i=1}^{n} x_{i}-\sum_{i=1}^{n} y_{i} \geq c \cdot \sqrt{n}$.

For appropriate $c$ one has $A D V(G)=\Omega(1$ ) (where $A D V(G)$ is defined like $A D V_{r}(G)$, but with regard to the uniform distribution over $\left.\{0,1\}^{2 n}\right)$. This happens, because a "large discrepancy" in $x$-sum and $y$ sum is more likely if we assume $\sum_{i=1}^{n} x_{i} \geq \frac{n}{2}$ and $\sum_{i=1}^{n} y_{i} \leq \frac{n}{2}$ than if we assume (say) $\sum_{i=1}^{n} x_{i} \geq \frac{n}{2}$ 
and $\sum_{i=1}^{n} y_{i} \geq \frac{n}{2}$. This phenomenon has been independently observed by Bultman [B].

Corollary 3.7 The class of boolean functions computable by constant size boolean threshold circuits of depth 2 with integer weights of polynomial size is properly contained in the class of boolean functions computable by constant size $\sigma$-circuits of depth 2 with polynomial size rational weights (even with common polynomial size denominator) and separation $\frac{1}{\text { poly }}$.

The same statement holds if one considers arbitrary real weights for both types of circuits (still with separation $\frac{1}{\text { poly }}$ ).

Proof. It is quite easy to simulate boolean threshold circuits of size $s$ and constant depth $d$ by sigmoid threshold circuits of the same size and depth. The containment is proper as a consequence of Theorems 3.1 and 2.2 .

\section{Simulation Results and Separation Boosting}

$T C_{d}^{0}(\gamma)$ is the class of those families $\left(g_{n} \mid n \in \mathrm{N}\right)$ of boolean functions that are computable, with separation $\Omega\left(\frac{1}{\text { poly }(n)}\right)$, by polynomial size, depth $d \gamma$-circuits whose weights are reals of absolute value at most poly $(n) . T C_{d}^{0}$ ([HMPST]) is the corresponding class of families of boolean functions computable by polynomial size, depth $d$ boolean threshold circuits whose weights are polynomial size integers.

Theorem 4.1 Let $\gamma: \mathbf{R} \rightarrow[0,1]$ be a nondecreasing function that is Lipschitz-bounded and converges fast to 0 (resp. 1) in the following sense:

\section{$\forall k \in \mathbf{N} \quad \exists l \in \mathbf{N} \quad \forall n \in \mathbf{N}^{+}$}

$$
\left(\gamma\left(-n^{l}\right) \leq \frac{1}{n^{k} n^{l}} \text { and } 1-\gamma\left(n^{l}\right) \leq \frac{1}{n^{k} n^{l}}\right) .
$$

Then the following holds.

(a) For every $d \in \mathbf{N}, T C_{d}^{0}=T C_{d}^{0}(\gamma)$.

(b) The class $T C_{d}^{0}(\gamma)$ does not change if we demand separation $\Omega(1)$.

Observe, that the above class of functions also includes the standard sigmoid $\sigma$.

Proof Sketch. Assume that $\left(g_{n} \mid n \in \mathrm{N}\right)$ is a family of boolean functions in $T C_{d}^{0}(\gamma)$. Thus $\left(g_{n} \mid n \in \mathrm{N}\right)$ can be computed with separation $\frac{1}{p(n)}$ by some family $\left(C_{n} \mid n \in \mathrm{N}\right)$ of $\gamma$-circuits of depth $d$ with the number of gates and the size of weights bounded by $q(n)$ (for some polynomials $p$ and $q$ ). Since $\gamma$ is Lipschitzbounded, and since the depth $d$ of $C_{n}$ is a constant, there exists a polynomial $r(n)$ with the following property:
If the gate function of each gate $G$ in $C_{n}$ is replaced by some arbitrary function $\gamma_{G}$ : $\mathbf{R} \rightarrow \mathbf{R}$ (where the functions $\gamma_{G}$ may be different for different gates $G$ ) such that

$$
\forall x \in \mathbf{R}\left(\left|\gamma(x)-\gamma_{G}(x)\right| \leq \frac{1}{r(n)}\right),
$$

then for each input $x_{1}, \ldots, x_{n}$ of $C_{n}$ the value of the output gate of the new circuit differs from the value of the output gate of $C_{n}$ by at most $\frac{1}{2 p(n)}$.

In order to construct a boolean threshold circuit $C_{n}^{b}$ that computes $g_{n}$, one replaces in $C_{n}$ each internal $\gamma$-gate that outputs $\gamma\left(\sum_{j=1}^{m} \alpha_{j} y_{j}-\theta\right)$ for inputs $y_{1}, \ldots, y_{m} \in[0,1]$ (with reals $\alpha_{1}, \ldots, \alpha_{m}, \theta$ of polynomial size in $n$ ) by a weighted sum

$$
S(\vec{y}):=\sum_{k=1}^{l} \frac{1}{2 r(n)} H_{k}\left(y_{1}, \ldots, y_{m}\right)
$$

of $l:=2 r(n)$ boolean threshold gates $H_{1}, \ldots, H_{l}$ (which use the same weights $\alpha_{1}, \ldots, \alpha_{m}$ as $G$ ). The function $S$ is chosen to be a step function which approximates $\gamma$ such that for all $y_{1}, \ldots, y_{m} \in[0,1]$,

$$
\left|\gamma\left(\sum_{j=1}^{m} \alpha_{j} y_{j}-\theta\right)-S(\vec{y})\right| \leq \frac{1}{2 r(n)}
$$

In a second step, one replaces each of the boolean threshold gates $H_{k}$ by a boolean threshold gate $H_{k}^{\prime}$ whose weights and thresholds are integers of polynomial size. We set

$$
S^{\prime}(\vec{y}):=\sum_{k=1}^{l} \frac{1}{2 r(n)} H_{k}^{\prime}\left(y_{1}, \ldots, y_{m}\right) .
$$

The threshold gates $H_{k}^{\prime}$ are chosen such that

$$
\forall y_{1}, \ldots, y_{m} \in[0,1]\left(\left|S(\vec{y})-S^{\prime}(\vec{y})\right| \leq \frac{1}{2 r(n)}\right) .
$$

Let $C_{n}^{\prime}$ be the circuit that results from $C_{n}$ by replacing in the described manner each internal $\gamma$-gate in $C_{n}$ by an array of boolean threshold gates $H_{k}^{\prime}$. For every input, the value of the output gates of $C_{n}$ and $C_{n}^{\prime}$ differ by at most $\frac{1}{2 p(n)}$. Hence we can replace the output gate of $C_{n}^{\prime}$ by a boolean threshold gate with integer weights and threshold of polynomial size such that the resulting boolean threshold circuit $C_{n}^{b}$ computes $g_{n}$. This shows that $\left(g_{n} \mid n \in \mathbf{N}\right) \in T C_{d}^{0}$.

In order to prove the other inclusion assume that $\left(g_{n} \mid n \in \mathbf{N}\right) \in T C_{d}^{0}$ is computed by a family $\left(B_{n} \mid n \in\right.$ N) of boolean threshold circuits of depth $d$, where $B_{n}$ has at most $p(n)$ gates and its weights and thresholds are integers of absolute value at most $q(n)$ (for some polynomials $p$ and $q$ ). 
Fix some constant $k \in N$ such that $4 p(n) \cdot q(n) \leq n^{k}$ for all $n$ with $n \geq 2$. According to our assumption about $\gamma$, there exists some $l \in \mathbf{N}$ such that

$$
\forall n \geq 1\left(\gamma\left(-n^{l}\right) \leq \frac{1}{n^{k} n^{l}} \text { and } 1-\gamma\left(n^{l}\right) \leq \frac{1}{n^{k} n^{l}}\right) .
$$

Let $B_{n}^{\prime}$ be a boolean threshold circuit that results from $B_{n}$ by multiplying first all weights and thresholds of gates in $B_{n}$ by $4 n^{l}$, and by lowering then each threshold by $2 n^{l}$. It is obvious that $B_{n}^{\prime}$ also computes the boolean function $g_{n}$. In addition, for each circuit input the weighted sum at each gate in $B_{n}^{\prime}$ has distance at least $2 n^{l}$ from its threshold.

Let $C_{n}$ be the $\gamma$-circuit that results if we replace each boolean threshold gate in $B_{n}^{\prime}$ by a $\gamma$-gate with the same weights and threshold. Then one shows by induction on the depth of a gate $G$ in $C_{n}$ that

for every boolean circuit input, the output of $G$ differs by at mast $\delta_{n}$ from the output of the corresponding gate in $B_{n}^{\prime}$, where $\delta_{n}:=\max \left(1-\gamma\left(n^{l}\right), \gamma\left(-n^{l}\right)\right)$.

In the induction step one exploits that by the choice of $k, l \in \mathbf{N}$ one has

$$
4 \cdot p(n) \cdot q(n) \cdot n^{l} \cdot \delta_{n} \leq n^{k} \cdot n^{l} \cdot \delta_{n} \leq 1 .
$$

This observation, together with the fact that the absolute value of each weight of a gate $G$ in $C_{n}$ is bounded by $q(n) \cdot 4 n^{l}$, implies that a change of at most $\delta_{n}$ in each of the at most $p(n)$ inputs of $G$ causes a change of at most one in the value of the weighted sum that reaches $G$. Hence for any boolean circuit input the weighted sum of $G$ has distance at least $2 n^{l}-1$ from its threshold. Therefore the output value of the $\gamma$-gate $G$ differs by at most $\max \left(1-\gamma\left(2 n^{l}-1\right), \gamma\left(-2 n^{l}+1\right)\right) \leq$ $\max \left(1-\gamma\left(n^{l}\right), \gamma\left(-n^{l}\right)\right)=\delta_{n}$ from the output of the corresponding boolean threshold gate in $B_{n}^{\prime}$.

The preceding argument implies that for any $n \geq 2$ the $\gamma$-circuit $C_{n}$ with outer threshold $\frac{1}{2}$ computes the boolean function $g_{n}$ with separation $\frac{1}{4}$.

Remark 4.2 One can also simulate polynomial size $\sigma$ circuits with weights of absolute value at most $2^{\text {poly(n) }}$ by polynomial size boolean threshold circuits with 0 1 weights; however in this case the circuit depth increases by a constant factor. This simulation can be extended to the case of real-valued inputs, where we assume that polynomially many bits of each real input are given as inputs to the simulating boolean threshold circuit.

\section{References}

[B] W.J. Bultman, "Topics in the theory of machine learning and neural computing", Ph.D Dissertation, University of Illinois at Chicago, 1991.

[HKP] J. Hertz, A. Krogh and R.G. Palmer, "Introduction to the Theory of Neural Computation", Addison-Wesley, Redwood City, 1991.
[HMPST] A.Hajnal, W. Maass, P. Pudlak, M. Szegedy and G. Turan, "Threshold Circuits of Bounded Depth", Proc. of the 28th Annual Symp. on Foundations of Computer Science, pp. 99-110, 1987. To appear in J. Comp. Syst. Sci.

[K] C.C. Klimasauskas, "The 1989 Neuro-Computing Bibliography", MIT Press, Cambridge, 1989.

[M] S. Muroga, "Threshold Logic and its Applications", Wiley, New York, 1971.

[MT] W. Maass and G. Turan, "How Fast Can a Threshold Gate Learn?", in: Computational Learning Theory and Natural Learning Systems: Constraints and Prospects, G. Drastal, S.J. Hanson and R. Rivest eds., MIT Press, to appear.

[S] E.L. Schwartz, "Computational Neuroscience", MIT Press, Cambridge, 1990.

[So] E.D. Sontag, "Feedforward Nets for Interpolation and Classification", J. Comp. Syst. Sci., to appear.

[SS1] E.D. Sontag and H.J. Sussmann, "Backpropagation can give rise to spurious local minima even for networks without hidden layers", Complex Systems 3, pp. 91-106, 1989.

[SS2] E.D. Sontag and H.J. Sussmann, "Backpropagation separates where Perceptrons do", Neural Networks, 4, pp. 243-249, 1991

[WK] S.M. Weiss, C.A. Kulikowsky, "Computer Systems that Learn", Morgan Kaufmann, San Mateo, 1991. 